\title{
CONVEX REARRANGEMENTS OF LÉVY PROCESSES
}

\author{
Youri Davydov ${ }^{1}$ and Emmanuel Thilly ${ }^{2}$
}

\begin{abstract}
In this paper we study asymptotic behavior of convex rearrangements of Lévy processes. In particular we obtain Glivenko-Cantelli-type strong limit theorems for the convexifications when the corresponding Lévy measure is regularly varying at $0^{+}$with exponent $\alpha \in(1,2)$.
\end{abstract}

Mathematics Subject Classification. 60G51, 60G52, 60G17.

Received March 13, 2006. Revised June 21, 2006.

\section{INTRODUCTION}

Let $\xi=\{\xi(t), t \geq 0\}$ be a stochastic process taking on real values and such that $\xi(0)=0$ almost surely. For any $i=1,2, \ldots$, let $X_{i}$ be the increment

$$
X_{i}=\xi(i)-\xi(i-1) .
$$

Given $n$, let $X_{1: n} \leq \ldots \leq X_{n: n}$ be the order statistics of $X_{1}, \ldots, X_{n}$. We connect the points $(0,0),\left(k, \sum_{i=1}^{k} X_{i: n}\right)$, $k=1,2, \ldots, n$, using straight lines and obtain the curve

$$
\mathrm{C}_{n}(x)= \begin{cases}\sum_{i=1}^{[x]} X_{i: n}+(x-[x]) X_{[x]+1: n}, & \text { if } 0 \leq x<n, \\ \sum_{i=1}^{n} X_{i: n}\left(=\sum_{i=1}^{n} X_{i}=\xi(n)\right), & \text { if } x=n .\end{cases}
$$

The function $\mathrm{C}_{n}$ is called the convex rearrangement of $\xi$ on the interval $[0, n]$, in view of the fact that it is convex on the interval. Since both the function $\mathrm{C}_{n}$ and its domain of definition depend on $n$, it is natural and convenient to redefine $\mathrm{C}_{n}$ as follows:

$$
\mathrm{C}_{n}^{0}(t)=\mathrm{C}_{n}(n t), \quad 0 \leq t \leq 1
$$

The function $\mathrm{C}_{n}^{0}$ possesses the geometric properties of the original one, $\mathrm{C}_{n}$. If, however, the stochastic process $\xi$ is defined on the compact interval $[0,1]$, then instead of the above $X_{i}$ 's we use the following increments

$$
X_{i, n}=\xi\left(\frac{i}{n}\right)-\xi\left(\frac{i-1}{n}\right)
$$

\footnotetext{
Keywords and phrases. Convex rearrangements, Lévy processes, strong laws, Lorenz curve, regularly varying functions.

1 Laboratoire Paul Painlevé - UMR 8524 université de Lille I, Bât. M2 59655, Villeneuve d'Ascq, France; youri.davydov@univ-lille1.fr

2 Laboratoire GREMARS, EA 2459 université de Lille 3, Maison de la recherche BP 149, 59653 Villeneuve d'Ascq, France; emmanuel.thilly@univ-lille3.fr 
to define both $\mathrm{C}_{n}$ and $\mathrm{C}_{n}^{0}$. The important difference between the present situation and the previous one is that the random variables $X_{i, n}$ are now dependent on $n$. When needed, in both $[0, \infty)$ and $[0,1]$ cases we use the notation $\mathrm{C}_{n}^{0}[\xi]$ to indicate the dependence of $\mathrm{C}_{n}^{0}$ on $\xi$. The following general definition of convex rearrangements is given in [8].

Definition 1.1. Let $f$ be a measurable real function on $[0,1]$. A function $T f$ is said to be the monotone rearrangement of $f$, if it is monotonic increasing, continuous from the right, and has the same distribution as $f$ it means that $\lambda f^{-1}=\lambda(T f)^{-1}$, where $\lambda$ denotes the Lebesgue measure. The operator $V$ defined on the set of absolutely continuous functions on $[0,1]$ by the formula

$$
V f(t)=f(0)+\int_{0}^{t} T\left(f^{\prime}\right)(s) \mathrm{d} s
$$

is said to be the operator of convex rearrangement.

It is evident that $V f$ is convex and takes on the same values at the endpoints of $[0,1]$ as the function $f$. Let $\xi=\{\xi(t), \quad t \in[0,1]\}$ and consider the sequences of polygonal approximations of $\xi$ determined by the uniform partitions of the interval $[0,1]$ as follows:

$$
\xi_{n}(t)=\xi\left(\frac{i}{n}\right)+\left(t-\frac{i}{n}\right) X_{i, n}, \quad t \in[i / n,(i+1) / n] .
$$

Then, for each $t \in[0,1]$,

$$
V \xi_{n}(t)=\mathrm{C}_{n}^{0}[\xi](t) .
$$

Recently, asymptotic behavior of $\mathrm{C}_{n}^{0}[\xi]$ has been established for various families of stochastic processes. For example, strong limit theorems have been obtained when $\xi$ is a random walk [13], strictly stable process [8], and Gaussian process [14]. Moreover, it has been noted in [11] that the asymptotic behavior of convexifications is closely related to the behavior of oscillations of smoothed processes, a topic extensively studied in [1] and [22]. It is worthwhile to note that the knowledge of asymptotic behaviour of $\mathrm{C}_{n}^{0}[\xi]$ gives useful information about the oscillations of the original process and can be used in various applications. It also serves as a starting point of a non-standard approach to the estimation problem of parameters of the process. For example, based on [11], a new method for estimating the Hurst index of self similar Gaussian processes has been proposed in [20]. Based on these considerations a statistical index for measuring the fluctuations of stochastic processes was proposed in [10]. In addition, a connection between convex rearrangements and econometric theory (cf. e.g., a survey paper [15] for more detail) was investigated in [14] by noting that the limiting curves in the aforementioned strong theorems are generalized Lorenz curves, which is a class of well known functions in econometrics. For an account of various properties of convex rearrangements, results, and applications, we refer to [15].

In the present work we study convex rearrangements when $\xi=\{\xi(t), t \in[0,1]\}$ is a Lévy process, which is, by definition, a stochastic process with stationary and independent increments. If the Gaussian component is absent, and if the Lévy measure - which appears in the characteristic function of the random variable $\xi(t)$ - is regularly varying with exponent $\alpha \in(1,2)$ at $0^{+}$, then we obtain a strong limit theorem ( $c f$. Th. 2.1 below). The limiting curve is non-random. More precisely, the limiting curve is a generalized Lorenz curve defined by a strictly stable random variable with exponent $\alpha \in(1,2)$. This result relates very nicely to the result of [8]. Intuitively, the result should not be surprising because the aforementioned condition of regular variation means that the process we consider is locally $\alpha$-stable.

We restrict ourselves to the study of the case $\alpha \in(1,2)$ only, due to the following reason. It is not difficult to see (cf., e.g., [8]) that almost sure convergence of $\mathrm{C}_{n}^{0}[\xi]$ is equivalent, loosely speaking, to the strong law of large numbers (SLLN) for triangular arrays of i.i.d. random variables that are generated by appropriately normalized increments of the process. This SLLN requires the existence of the first moment. Hence, $\alpha$ must be larger than 1. If $\alpha \leq 1$, we cannot have, in principle, more than weak convergence. We refer to [13] and [9] for detail, where this type of convergence has been studied for convexifications of i.i.d. sequences. Even though 
such results are not too difficult to obtain, they are beyond the scope of the present work. Other possible topics of interest include establishing rates of convergence. In some cases, the central limit theorem for $\mathrm{C}_{n}^{0}[\xi]$ has been obtained. For example, in [14] we find a CLT in the case of the fractional Brownian motion. We also refer to [23] for related results for increments of smoothed Levy processes and to [4-7] for the rate of convergence to Lorenz curve in the i.i.d. case. All these extensions and generalizations are interesting research topics, but they are beyond the scope of the present paper.

The rest of this papers is organized as follows. In Section 2 we state and discuss the main result, which is Theorem 2.1. In Section 3 we formulate and prove a number of lemmas that play crucial roles in establishing Theorem 2.1. The proof of Theorem 2.1 itself is given in the second half of Section 3.

\section{MAin Result}

Let $\xi=\{\xi(t), t \in[0,1]\}$ be a Lévy process defined on a probability space $(\Omega, \mathcal{A}, \mathrm{P})$ and taking on real values. The Lévy-Khintchine representation for the characteristic function $\Phi_{t}$ of the random variable $\xi(t)$ can be written as (cf., e.g., [2] p. 11)

where

$$
\Phi_{t}(\theta)=\exp \{-t \Psi(\theta)\}, \quad \theta \in \mathrm{R},
$$

$$
\Psi(\theta)=-i \gamma \theta+\frac{1}{2} \sigma^{2} \theta^{2}+\int_{\mathrm{R}}\left(1-e^{i \theta u}+i \theta u \mathbb{I}_{(-1,1)}(u)\right) \Pi(\mathrm{d} u)
$$

with $\gamma \in \mathrm{R}$ and $\sigma \in \mathrm{R}^{+}:=[0,+\infty[$. The $\Pi$ in the formula above is a Borel measure on the real line such that, $\Pi(\{0\})=0, \Pi(\{u: u \geq a\})<\infty$ for every $a>0$, and $\int_{|u|<1} u^{2} \Pi(\mathrm{d} u)<\infty$. Assume also that $\int_{|u|>1}|u| \Pi(\mathrm{d} u)<$ $\infty$. From Theorem 8 in [21], the above assumptions ensure that $\mathrm{E}|\xi(1)|<\infty$. We still need to introduce additional notation. Let

$$
G(u):=\Pi(u,+\infty)+\Pi(-\infty,-u), \quad u \in \mathrm{R}^{+} .
$$

Moreover, let $f^{-1}$ be the generalized inverse of a monotone function $f$, that is, $f^{-1}(u)=\inf \{t: f(t) \geq u\}$. For the Lévy process $\xi=\{\xi(t), t \in[0,1]\}$ we impose the following assumptions:

$\mathbf{H}$ : There exists an $\alpha \in(1,2)$, a slowly varying function $\ell$ at the origin and functions $C^{+}(u), C^{-}(u)$ such that:

- $\Pi(u,+\infty)=C^{+}(u) u^{-\alpha} \ell(u)$

- $\Pi(-\infty,-u)=C^{-}(u) u^{-\alpha} \ell(u)$;

- $C^{+}(u) \rightarrow C^{+}$when $\quad u \downarrow 0$;

- $C^{-}(u) \rightarrow C^{-}$when $\quad u \downarrow 0$;

- $C^{+}+C^{-}=1$.

Theorem 2.1. Let $\xi=\{\xi(t), t \in[0,1]\}$ be a Lévy process satisfying assumption $\mathbf{H}$. If $\sigma=0$, then for all $t \in[0,1]$ we have

$$
\lim _{n \rightarrow \infty} \frac{1}{a_{n}} \mathrm{C}_{n}^{0}[\xi](t)=\mathrm{L}_{\alpha}^{(1)}(\mathrm{t})
$$

almost surely, where $a_{n}:=n G^{-1}(n)$ and the limit $\mathrm{L}_{\alpha}^{(1)}(\mathrm{t}):=\int_{0}^{\mathrm{t}} F_{\alpha}^{-1}(\mathrm{~s}) \mathrm{ds}$ is the Lorenz curve corresponding to the distribution $F_{\alpha}$ of a strictly $\alpha$-stable random variable $(1<\alpha<2)$ whose characteristic function is

$$
\phi_{\alpha}(\theta):=\exp \left\{\frac{i\left(C^{-}-C^{+}\right) \alpha}{\alpha-1} \theta-\int_{-\infty}^{+\infty}\left(1-e^{i u \theta}+i u \theta \mathbb{I}_{(-1,1)}(u)\right) \mathcal{L}_{\alpha}(\mathrm{d} u)\right\}
$$

with $\mathcal{L}_{\alpha}(\mathrm{d} u)=\alpha|u|^{-\alpha-1}\left(C^{+} \mathbb{I}_{\{u>0\}}+C^{-} \mathbb{1}_{\{u<0\}}\right)$. If, however, $\sigma \neq 0$, then for all $t \in[0,1]$ we have that

$$
\lim _{n \rightarrow \infty} \frac{1}{\sqrt{n}} \mathrm{C}_{n}^{0}[\xi](t)=\mathrm{L}_{\sigma}^{(2)}(\mathrm{t})
$$


almost surely, where $\mathrm{L}_{\sigma}^{(2)}(\mathrm{t}):=\int_{0}^{\mathrm{t}} \Phi_{\sigma}^{-1}(\mathrm{~s}) \mathrm{ds}$ with $\Phi_{\sigma}$ denoting the centered normal distribution with the variance $\sigma^{2}$.

Actually, Theorem 2.1 implies stronger statements than those in (2.1) and (2.3). Namely, since for each $n \geq 1$ the function $\mathrm{C}_{n}^{0}[\xi](t)$ is convex, any of the two statements (2.1) and (2.3) implies that the convergence is, in fact, uniform in $t \in[0,1]$.

\section{Proof of Theorem 2.1}

Consider the decomposition of $\xi_{t}$ as a sum of independent processes (cf., e.g., [18] p. 258 for more details):

$$
\xi_{t}=\gamma t+\sigma W_{t}+Y_{t}
$$

where $Y_{t}=\int_{|x| \geq 1} x \nu_{t}(\mathrm{~d} x)+\int_{|x|<1} x \nu_{t}^{*}(\mathrm{~d} x), W$ is a standard Wiener process, $\left(\nu_{t}(\mathrm{~d} x), t \geq 0\right)$ is the Poisson random measure of discontinuities, and $\nu_{t}^{*}=\nu_{t}(B)-t \Pi(B)$ for every Borel set $B$ on the real line such $0 \notin B$. Moreover $\left\{W_{t}, t \geq 0\right\}$ and $\left\{\nu_{t}(\cdot), t \geq 0\right\}$ are independent.

Now we recall the following result proved in [12]. If $X_{t}$ is a stochastic process such that $\mathrm{C}_{n}^{0}[X](t)$ converges to some limit $L(t)$ and if $f$ is an absolutely continuous function on $[0,1]$, then $\mathrm{C}_{n}^{0}[f+X](t)$ also converges to $L(t)$. That implies that we can assume $\gamma$ to be zero without loss of generality when studying the convexifications of $\xi$. Consequently throughout the rest of this section we assume $\gamma=0$.

\subsection{Preliminary results}

Lemma 3.1. Let $g$ be a function defined on $[0,1]$ and taking on non-negative values. Furthermore, let $g$ be regularly varying at $0^{+}$with exponent $\rho$. Then we have the following two statements:

1. If $\rho<-1$ or if $\rho=-1$ and $\int_{0}^{1} g(x) \mathrm{d} x=\infty$, then we have that $y g(y) / \int_{y}^{1} g(x) \mathrm{d} x \longrightarrow-(\rho+1)$, when $y \rightarrow 0$.

2. If $\rho>-1$ or if $\rho=-1$ and $\int_{0}^{1} g(x) \mathrm{d} x<\infty$, then we have $y g(y) / \int_{0}^{y} g(x) \mathrm{d} x \longrightarrow(\rho+1)$, when $y \rightarrow 0$.

Proof. See Section VIII. 9, p. 280, in [17].

Lemma 3.2. Let $\Theta$ be an arbitrary set, and let $\left\{\zeta_{\theta}\right\}_{\theta \in \Theta}$ be a family of random variables defined on a probability space $(\Omega, \mathcal{B}, \mathrm{P})$. For each $\theta \in \Theta$, let $\zeta_{\theta}^{1}$ and $\zeta_{\theta}^{2}$ be two independent copies of $\zeta_{\theta}$. Put $\widetilde{\zeta}_{\theta}:=\zeta_{\theta}^{1}-\zeta_{\theta}^{2}$ (symmetrized version of $\left.\zeta_{\theta}\right)$. Suppose that

1. $\left\{\widetilde{\zeta}_{\theta}\right\}_{\theta \in \Theta}$ is uniformly integrable, and

2. $\sup _{\theta \in \Theta} \mathrm{P}\left(\left|\zeta_{\theta}\right|>a\right) \longrightarrow 0$ when $a \rightarrow+\infty$.

Then the family $\left\{\zeta_{\theta}\right\}_{\theta \in \Theta}$ is uniformly integrable.

Proof. The proof is quite easy by dealing with standard arguments around uniformly integrablility.

Lemma 3.3. Let $F$ be a symmetric probability distribution with characteristic function $\phi(t), t \in \mathrm{R}$. For any $\lambda \in(0,2)$ and for all $C>0$, we have the bound

$$
\int_{|x|>C}|x|^{\lambda} F(\mathrm{~d} x) \leq \frac{1}{Q(1)} \int_{0}^{1 / C} t^{-\lambda-1}[1-\phi(t)] \mathrm{d} t,
$$

where $Q(u)=\int_{0}^{u} y^{-\lambda-1}(1-\cos y) \mathrm{d} y$.

Proof. The proof is a direct adaptation of proof of Theorem 5. $a$ in [21]. 


\subsection{Case $\sigma=0$}

Lemma 3.4. Let $\xi=\{\xi(t), t \geq 0\}$ be a Lévy process such that $\gamma=\sigma=0$, and let $\mathbf{H}$ hold. We have that

$$
\frac{1}{b(t)} \xi(t) \Longrightarrow \eta_{\alpha} \quad \text { when } \quad t \rightarrow 0
$$

where $\Longrightarrow$ denotes weak convergence, $b(t):=G^{-1}(1 / t)$, and $\eta_{\alpha}$ is a strictly $\alpha$-stable random variable with $1<\alpha<2$. Furthermore, we have that

$$
\mathrm{E}\left(\frac{1}{b(t)} \xi(t)\right) \longrightarrow \mathrm{E}\left(\eta_{\alpha}\right) \quad \text { when } \quad t \rightarrow 0
$$

where $\mathrm{E}$ denotes the mathematical expectation on $(\Omega, \mathcal{A}, \mathrm{P})$.

Before proving Lemma 3.4, we make a comment on statement (3.3). Namely, we note that in [16] necessary and sufficient conditions in term of the characteristic function of the Lévy process are given for having the convergence of suitably normalized $X(t)$ to a normal distribution when $t$ goes to zero. Moreover note also that (3.3) is an immediate consequence of Theorem 1 in [22] where Case 3 corresponds exactly to $\sigma=0$ and $\mathbf{H}$.

Proof of statement (3.3). The characteristic function of the random variable $\xi(t) / b(t)$ is given by

$$
\Phi_{t}\left(\frac{\theta}{b(t)}\right)=\exp \left\{-t \int_{\mathrm{R}}\left(1-\exp i u \frac{\theta}{b(t)}+i u \frac{\theta}{b(t)} \mathbb{I}_{(-1,1)}(u)\right) \Pi(\mathrm{d} u)\right\} .
$$

The change of variable $y=u / b(t)$ gives

$$
\Phi_{t}\left(\frac{\theta}{b(t)}\right)=\exp \left\{-\psi_{t}(\theta)\right\}
$$

where

$$
\psi_{t}(\theta)=\int_{\mathrm{R}}\left(1-\mathrm{e}^{i \theta y}+\mathbb{I}_{\{|y|<1\}}\right) \mathcal{P}_{t}(\mathrm{~d} y)+i \theta \int_{\mathrm{R}} y \mathbb{I}_{\{1<|y|<1 / b(t)\}} \mathcal{P}_{t}(\mathrm{~d} y),
$$

and $\mathcal{Q}_{t}:=\Pi g_{t}^{-1}$ with $g_{t}(u):=u / b(t)$ and $\mathcal{P}_{t}:=t \mathcal{Q}_{t}$.

Hence, statement (3.3) will be proved as soon as we show that for each $\theta \in \mathrm{R}$, the quantity $\Phi_{t}\left(\frac{\theta}{b(t)}\right)$ converges to $\phi_{\alpha}(\theta)$ defined in (2.2) when $t$ goes to zero. For any fixed $t \in[0,1]$, it is well known that the law of the random variable $\xi_{t}$ is infinitely divisible. Hence, from a well known criterion about infinitely divisible distributions $(c f$. e.g. [19], Th. 5.6.2 and Prop. 5.7.4), we have (3.3) if we establish the following three statements:

i) For every $\varepsilon>0$ we have $\mathcal{P}_{t}^{\varepsilon} \Longrightarrow \mathcal{L}_{\alpha}^{\varepsilon}$, when $t \rightarrow 0$, where $\mathcal{P}_{t}^{\varepsilon}$ is the restriction of $\mathcal{P}_{t}$ to $\{u:|u|>\varepsilon\}$.

ii) $\int_{\mathrm{R}} y \mathbb{I}_{\{1<|y|<1 / b(t)\}}(y) \mathcal{P}_{t}(\mathrm{~d} y) \longrightarrow \int_{\mathrm{R} \backslash(-1,1)} y \mathcal{L}_{\alpha}(\mathrm{d} y)$, when $t \rightarrow 0$.

iii) $\lim _{r \downarrow 0}\left\{\limsup _{t \downarrow 0} \int_{\{|y|<r\}} y^{2} \mathcal{P}_{t}(\mathrm{~d} y)\right\}=0$.

We shall first prove i). Clearly, hypothesis $\mathbf{H}$ implies that $G(u) \sim u^{-\alpha} l(u)$ when $u$ is sufficiently small. Define

$$
\mathcal{G}_{\mathcal{P}_{t}}(u):= \begin{cases}\mathcal{P}_{t}([u, \infty)) & \text { if } u>0 \\ \mathcal{P}_{t}((-\infty, u]) & \text { if } u<0\end{cases}
$$

If $u>0$ is small, then

$$
\left.\mathcal{G}_{\mathcal{P}_{t}}(u)=\mathcal{P}_{t}[u, \infty)\right)=t \Pi\left(g_{t}^{-1}([u, \infty))\right)=C^{+} u^{-\alpha} \frac{\ell\left(u G^{-1}(1 / t)\right)}{\ell\left(G^{-1}(1 / t)\right)}
$$


Since $G^{-1}(1 / t) \longrightarrow 0$ when $t \downarrow 0$, and since $\ell$ is a slowly varying function at $0^{+}$then

$$
\frac{\ell\left(u G^{-1}(1 / t)\right)}{\ell\left(G^{-1}(1 / t)\right)} \longrightarrow 1 \quad \text { when } \quad t \rightarrow 0 .
$$

Consequently,

$$
\mathcal{G}_{\mathcal{P}_{t}}(u) \longrightarrow C^{+} u^{-\alpha}, \quad t \downarrow 0 .
$$

Similar calculations yields that if $u<0$ is sufficiently close to zero, then

$$
\mathcal{G}_{\mathcal{P}_{t}}(u) \longrightarrow C^{+}|u|^{-\alpha}, \quad t \downarrow 0 .
$$

From statements (3.6) and (3.7) we deduce that for all $u$ in a neighborhood of 0 ,

$$
\mathcal{G}_{\mathcal{P}_{t}}(u) \longrightarrow\left(C^{+} \mathbb{1}_{\{u>0\}}+C^{-} \mathbb{1}_{\{u<0\}}\right)|u|^{-\alpha}, \quad t \rightarrow 0 .
$$

This yields condition $i$ ).

Now we shall show that $i$ ) holds. One easily verifies using the change of variable and the integration by parts formulas that

$$
\int_{1}^{1 / b(t)} y \mathcal{P}_{t}(\mathrm{~d} y)=\frac{t}{b(t)}\left\{\Pi([1, \infty))-b(t) \Pi([b(t), \infty))-\int_{b(t)}^{1} \Pi([u, \infty)) \mathrm{d} u\right\} .
$$

As the generalized inverse of a regularly varying function with exponent $\alpha$ is also a regularly varying function with exponent $1 / \alpha(c f .$, e.g., $[3]$ p. 17 , for more details), there exists a slowly varying function $\widetilde{\ell}$ such that $b(t)=G^{-1}(1 / t)=t^{-1 / \alpha} \widetilde{\ell}(t)$. This yields

$$
\frac{t}{b(t)} \Pi([1, \infty))=C^{+} \ell(1) \frac{t}{b(t)}=C^{+} \ell(1) t^{1-1 / \alpha} \widetilde{\ell}(t) \longrightarrow 0, \quad t \downarrow 0 .
$$

In addition, by properties of slowly varying functions we easily obtain that

$$
t \cdot \Pi([b(t), \infty)) \longrightarrow C^{+}, \quad t \downarrow 0 .
$$

Moreover, from the first part of Lemma 3.1 we obtain that

$$
\int_{b(t)}^{1} \Pi([u, \infty)) \mathrm{d} u=C^{+} \int_{b(t)}^{1} u^{-\alpha} \ell(u) \mathrm{d} u \sim \frac{C^{+}}{\alpha-1}(b(t))^{1-\alpha} \ell(b(t)), \quad t \downarrow 0 .
$$

Hence, we have

$$
\frac{t}{b(t)} \int_{b(t)}^{1} \Pi([u, \infty)) \mathrm{d} u \sim \frac{C^{+}}{\alpha-1} .
$$

In view of (3.9), (3.10) and (3.11), we deduce from (3.8) that

$$
\int_{1}^{1 / b(t)} y \mathcal{P}_{t}(\mathrm{~d} y) \sim-C^{+}-\frac{C^{+}}{\alpha-1}=-\frac{\alpha C^{+}}{\alpha-1}, \quad t \downarrow 0 .
$$

Similar calculations give

$$
\int_{-1 / b(t)}^{-1} y \mathcal{P}_{t}(\mathrm{~d} y) \sim \frac{\alpha C^{-}}{\alpha-1}, \quad t \downarrow 0 .
$$


Consequently,

$$
\int_{\mathrm{R}} y \mathbb{I}_{\{1<|y|<1 / b(t)\}} \mathcal{P}_{t}(\mathrm{~d} y) \sim \frac{\alpha\left(C^{-}-C^{+}\right)}{\alpha-1}, \quad t \downarrow 0 .
$$

This completes the proof of $i i)$.

To prove $i i i$ ), let $r>0$. Then using integration by parts we get

$$
\int_{0}^{r} y^{2} \mathcal{P}_{t}(\mathrm{~d} y)=\operatorname{tr}^{2} \Pi([r b(t), \infty))-2 t(b(t))^{-2} \int_{0}^{r b(t)} u \Pi([u, \infty)) \mathrm{d} u,
$$

Note that

$$
\operatorname{tr}^{2} \Pi([r b(t), \infty)) \sim C^{+} r^{2-\alpha}, \quad t \downarrow 0 .
$$

Using Lemma 3.1, we obtain that

$$
2 t(b(t))^{-2} \int_{0}^{r b(t)} u \Pi([u, \infty)) \mathrm{d} u \sim \frac{2 C^{+}}{2-\alpha} r^{2-\alpha}, \quad t \downarrow 0 .
$$

Consequently,

$$
\int_{0}^{r} y^{2} \mathcal{P}_{t}(\mathrm{~d} y) \sim C^{+}\left(\frac{\alpha}{\alpha-2}\right) r^{2-\alpha}
$$

Similar arguments lead to the statement

$$
\int_{-r}^{0} y^{2} \mathcal{P}_{t}(\mathrm{~d} y) \sim-C^{-}\left(\frac{\alpha}{\alpha-2}\right) r^{2-\alpha}
$$

Hence, for every $r>0$, we have that

$$
\int_{|y|<r} y^{2} \mathcal{P}_{t}(\mathrm{~d} y) \sim\left(C^{+}-C^{-}\right)\left(\frac{\alpha}{\alpha-2}\right) r^{2-\alpha} .
$$

Statement (3.12) and the fact that $\alpha \in(1,2)$ yield $i i i)$. Taking all these pieces together, we complete the proof of statement (3.3).

Proof of statement (3.4). In view of statement (3.3), it suffices to show that $\left\{\frac{1}{b(t)} \xi(t)\right\}_{t \in[0,1]}$ is uniformly integrable. We first do it in the symmetric case. Then we have

$$
\psi_{t}(\theta)=\int_{\mathrm{R}}(1-\cos \theta y) \mathcal{P}_{t}(\mathrm{~d} y)
$$

Let $\mathcal{P}_{\xi(t) / b(t)}$ be the probability distribution of the random variable $\frac{\xi(t)}{b(t)}$. By Lemma 3.3 we have that, for all $A>0$,

$$
\int_{A}^{\infty}|x| \mathcal{P}_{\xi(t) / b(t)}(\mathrm{d} x) \leq \frac{1}{Q(1)} \int_{0}^{1 / A} \theta^{-2}\left(1-\Phi_{t}(\theta)\right) \mathrm{d} \theta
$$

Fix $t \in[0,1]$. Then for each $\theta \in \mathrm{R}$ we have

$$
1-\Phi_{t}(\theta)=\frac{1-\left|\Phi_{t}(\theta)\right|^{2}}{1+\left|\Phi_{t}(\theta)\right|} \leq 1-\left|\Phi_{t}(\theta)\right|^{2} .
$$


However, if $1 / 2 \leq a \leq 1$, we have $1-a \leq-\log a$. Therefore for $\theta$ close to 0 and such that $\left|\Phi_{t}(\theta)\right|^{2} \geq 1 / 2$, we have the bound

$$
1-\left|\Phi_{t}(\theta)\right|^{2} \leq-2 \log \left|\Phi_{t}(\theta)\right| .
$$

Consequently, if $A$ is large enough, then we have that

$$
\int_{A}^{\infty}|x| \mathcal{P}_{\xi(t) / b(t)}(\mathrm{d} x) \leq \frac{2}{Q(1)} \int_{0}^{1 / A} \theta^{-2} \psi_{t}(\theta) \mathrm{d} \theta
$$

By the change of variables formula and the Fubini's theorem, we get that

$$
\int_{0}^{1 / A} \theta^{-2} \psi_{t}(\theta) \mathrm{d} \theta=\int_{0}^{1 / A}\left[\int_{\mathrm{R}} \frac{(1-\cos \theta y)}{\theta^{2}}\right] \mathcal{P}_{t}(\mathrm{~d} y) \mathrm{d} \theta=\int_{\mathrm{R}} y h\left(\frac{y}{A}\right) \mathcal{P}_{t}(\mathrm{~d} y),
$$

where $h(u)=\int_{0}^{u} \frac{1-\cos \theta}{\theta^{2}} \mathrm{~d} \theta$ for all $u \in \mathrm{R}$. Observe the following three properties:

a) $h(x) \longrightarrow 0$ when $x \rightarrow 0$;

b) there exists $K$ such that $0 \leq h(x) \leq K$ for all $x \in \mathrm{R}^{+}$;

c) there exists $K^{\prime}$ such that $h(x) \leq K^{\prime}|x|$ for all $x \in \mathrm{R}$.

Now, for all sufficiently large $A>0$ we have the bound

$$
\begin{aligned}
\int_{0}^{1 / A} \theta^{-2} \psi_{t}(\theta) \mathrm{d} \theta & =\int_{|y| \leq \sqrt{A}} y h\left(\frac{y}{A}\right) \mathcal{P}_{t}(\mathrm{~d} y)+\int_{|y|>\sqrt{A}} y h\left(\frac{y}{A}\right) \mathcal{P}_{t}(\mathrm{~d} y) \\
& =: \quad \mathrm{I}_{1}+\mathrm{I}_{2} .
\end{aligned}
$$

Property c) gives the bound $\quad \mathrm{I}_{1} \leq \frac{K^{\prime}}{A} \int_{|y|<\sqrt{A}} y^{2} \mathcal{P}_{t}(\mathrm{~d} y)$. Applying (3.12) with $r=\sqrt{A}$, we get that, when $t$ is sufficiently small,

$$
\mathrm{I}_{1} \leq K^{\prime \prime}\left(C^{+}-C^{-}\right)\left(\frac{\alpha}{\alpha-2}\right) A^{-\alpha / 2}
$$

where $K^{\prime \prime}>0$ is a constant. Because $\alpha>0$, we have

$$
\sup _{t \in[0,1]} \mathrm{I}_{1} \longrightarrow 0 \quad \text { when } \quad A \rightarrow \infty
$$

In addition, from property b) we deduce that

$$
\mathrm{I}_{2} \leq 2 K \int_{y>\sqrt{A}} y \mathcal{H}_{t}(\mathrm{~d} y)
$$

where $\mathcal{H}_{t}(u)=t \mathcal{Q}([u, \infty))=t \Pi([u b(t), \infty))$. Consequently, we have the equality

$$
\int_{y>\sqrt{A}} y \mathcal{H}_{t}(\mathrm{~d} y)=\frac{t}{b(t)} \int_{\sqrt{A} / b(t)}^{\infty} u \Pi(\mathrm{d} u) .
$$

Similar calculations as in (3.9) allow us to conclude that

$$
\sup _{t \in[0,1]} \mathrm{I}_{2} \longrightarrow 0 \quad \text { when } \quad A \rightarrow \infty
$$


From (3.14), (3.15), (3.16) and (3.18) we deduce that

$$
\sup _{t \in[0,1]} \int_{|\xi(t) / b(t)|>A}\left|\frac{\xi(t)}{b(t)}\right| \mathrm{dP} \longrightarrow 0, \quad \text { when } \quad A \rightarrow \infty .
$$

Hence, $\left\{\frac{1}{b(t)} \xi(t)\right\}_{t \in[0,1]}$ is uniformly integrable. This completes our proof of statement (3.4) in the symmetric case.

Now we remove the assumption about the symmetry of the law of $\xi(1)$. The weak convergence established in (3.3) shows that if $t_{0}$ is sufficiently small, then

$$
\sup _{0 \leq t \leq t_{0}} \mathrm{P}\left(\left|\frac{\xi(t)}{b(t)}\right|>a\right) \longrightarrow 0 \quad \text { when } \quad a \rightarrow \infty .
$$

Consequently, from Lemma 3.2 we conclude the proof of statement (3.4). In order to formulate our next lemma, we need the following definition.

Definition 3.1. We say that a sequence $\left\{g_{n}\right\}_{n \geq 1}$ of measurable functions on $[0,1]$ converges weakly on $\left([0,1], \mathcal{B}_{[0,1]}, \lambda\right)$ to $g$ if $\lambda g_{n}{ }^{-1} \Longrightarrow \lambda g^{-1}$. We shall write this as $g_{n} \stackrel{[0,1]}{\Longrightarrow} g$.

The next result is proved in [8].

Lemma 3.5. Let $\left\{f_{n}\right\}_{n \geq 1}$ be a sequence of absolutely continuous functions. Then the following statements are equivalent:

A1. There exists a convex function $f$ such that $V f_{n}(t) \underset{n \rightarrow \infty}{\longrightarrow} f(t), \forall t \in[0,1]$.

A2. The limit $a=\lim _{n \rightarrow \infty} f_{n}(0)$ exists and is finite. Furthermore there exists a function $g$ such that

a) $f_{n}^{\prime} \stackrel{[0,1]}{\Rightarrow} g$;

b) $\int_{0}^{1}\left(f_{n}^{\prime}\right)_{ \pm} \mathrm{d} \lambda \underset{n \rightarrow \infty}{\longrightarrow} \int_{0}^{1} g_{ \pm} \mathrm{d} \lambda$.

In addition, $a=f(0)$ holds and one can take $g=f^{\prime}$.

By applying this result to the sequences of polygonal approximations of $\xi$ we follow the method proposed in [8] where it is noted that $A 1$ and $A 2$ can be reduced to LLN-type results for appropriately normalized increments of the process under considerations. Indeed, for this we have to first show that

$$
\left(1 / a_{n}\right)\left(V \xi_{n}\right)^{\prime} \stackrel{[0,1]}{\Rightarrow} F_{\alpha}^{-1} \quad \text { a.s. }
$$

and then verify the convergence

$$
\frac{1}{a_{n}} \int_{0}^{1}\left(V \xi_{n}\right)_{ \pm}^{\prime} \mathrm{d} \lambda \longrightarrow \int_{0}^{1}\left(F_{\alpha}^{-1}\right)_{ \pm} \mathrm{d} s
$$

For statement (3.19) note that the distribution of $\xi_{n}^{\prime}$ and $T \xi_{n}^{\prime}$ coincide. Hence, it is sufficient to prove that

$$
\left(1 / a_{n}\right)\left(\xi_{n}^{\prime}\right) \stackrel{[0,1]}{\Rightarrow} F_{\alpha}^{-1} \quad \text { a.s. }
$$

Consider now the Fourier transform $f_{n}$ of the measure $\lambda\left(\left(1 / a_{n}\right) \xi_{n}^{\prime}\right)^{-1}$. We have

$$
f_{n}(t)=\frac{1}{n} \sum_{i=1}^{n} h_{1}\left(\beta_{n j}\right)+i \frac{1}{n} \sum_{i=1}^{n} h_{2}\left(\beta_{n j}\right),
$$


where $\beta_{n j}=n X_{j, n} / a_{n}$ for all $j=1, \ldots, n$, and $\quad h_{1}(x)=\cos (t x), \quad h_{2}(x)=\sin (t x)$. Similarly, the integrals in (3.20) are transformed into the sums $\frac{1}{n} \sum_{j=1}^{n} h\left(\beta_{n j}\right)$ with $h(x)=x_{ \pm}$. For any $n \geq 1$, the random variables $\left\{\beta_{n j}, \quad j=1, \ldots, n: \quad n \in \mathrm{N}\right\}$ are independent identically distributed. However, the rows of the array are not independent. This is why we cannot apply the standard strong law of large numbers. Consequently, Theorem 2.1 will be proved with the help of the following lemma.

Lemma 3.6. Consider the triangular array $\beta_{n i}=X_{i, n} / G^{-1}(n)$, where $i \in\{1, \ldots, n\}$. Let $h$ be a continuous function such that

$$
|h(s)| \leq C_{1}|s|+C_{2}
$$

with two positive constants $C_{1}$ and $C_{2}$. Then we have that with probability 1 ,

$$
\frac{1}{n} \sum_{i=1}^{n} h\left(\beta_{n i}\right) \longrightarrow \mathrm{E} h\left(\eta_{\alpha}\right), \quad n \rightarrow \infty .
$$

Proof. Put $D_{n}=n^{\delta}, \delta \in(1 / \alpha, 1), h_{n}(s)=h(s) 1_{\left[0, D_{n}\right]}(|h(s)|)$ and $\hat{h}_{n}(s)=h_{n}(s)-\mathrm{E}\left(h_{n}\left(\beta_{n 1}\right)\right)$. Obviously,

$$
\frac{1}{n} \sum_{i=1}^{n} h\left(\beta_{n i}\right)=\Sigma_{1}+\Sigma_{2}+\Sigma_{3}
$$

where $\Sigma_{1}=\frac{1}{n} \sum_{i=1}^{n} \hat{h}_{n}\left(\beta_{n i}\right), \Sigma_{2}=\frac{1}{n} \sum_{i=1}^{n} \mathrm{E} h_{n}\left(\beta_{n i}\right), \Sigma_{3}=\frac{1}{n} \sum_{i=1}^{n}\left(h\left(\beta_{n i}\right)-h_{n}\left(\beta_{n i}\right)\right)$.

Since for each $i \in\{1, \ldots, n\}$ the random variables $\beta_{n i}$ and $\beta_{n 1}$ are equal in law by the definition of $h_{n}$, we deduce from statement (3.4) that

$$
\Sigma_{2} \longrightarrow \mathrm{E} h\left(\eta_{\alpha}\right) \quad \text { when } n \rightarrow \infty \text {. }
$$

Consider the sum $\Sigma_{1}$. By definition of $h_{n}$ for all $x \in \mathrm{R}$ we have the bound $\left|h_{n}(x)\right| \leq n^{\delta}$. Consequently, $\mathrm{E}\left|h_{n}(x)\right| \leq n^{\delta}$, and so we have the bound

$$
\left|\hat{h}_{n}(x)\right| \leq 2 n^{\delta} .
$$

Now put $\tau_{i}:=\hat{h}_{n}\left(\beta_{n i}\right)$. Applying the Rosenthal's inequality with power $2 m$ to the sequence $\left(\tau_{i}\right)_{i}$ we obtain that there exists a constant $K_{m}>0$ such that

$$
\mathrm{E}\left|\Sigma_{1}\right|^{2 m} \leq K_{m}\left\{\frac{1}{n^{2 m-1}} \mathrm{E}\left|\tau_{1}\right|^{2 m}+\frac{1}{n^{m}}\left(\mathrm{E}\left|\tau_{1}\right|^{2}\right)^{m}\right\} .
$$

Since $\mathrm{E}|\xi(1)|<\infty$, we have $\mathrm{E}\left|\tau_{1}\right|<\infty$. Consequently, by bound (3.24) there exists a constant $M_{1}>0$ such that

$$
\mathrm{E}\left|\tau_{1}\right|^{2}=\mathrm{E}\left|\tau_{1}\right| \cdot\left|\tau_{1}\right| \leq 2 \mathrm{E}\left|\tau_{1}\right| n^{\delta}<M_{1} n^{\delta} .
$$

Similar argument gives the existence of a constant $M_{2}>0$ such that

$$
\mathrm{E}\left|\tau_{1}\right|^{2 m} \leq M_{2} n^{(2 m-1) \delta} .
$$

From bound (3.25) we deduce that

$$
\mathrm{E}\left|\Sigma_{1}\right|^{2 m} \leq K_{m}\left\{M_{2} \cdot n^{(2 m-1)(\delta-1)}+M_{1} \cdot n^{m(\delta-1)}\right\} .
$$

Since $\delta<1$ for all sufficiently large $m$, it follows that $\mathrm{E}\left|\Sigma_{1}\right|^{2 m} \leq K_{m}^{\prime} n^{-\kappa}$, where $K_{m}^{\prime}>0$ is a constant and $\kappa>1$. Using the Borel-Cantelli lemma in a standard way, we obtain that $\Sigma_{1} \longrightarrow 0$, a.s.

Now we consider $\Sigma_{3}$. Evidently, $\Sigma_{3}=\Sigma_{3}^{+}+\Sigma_{3}^{-}$, where

$$
\Sigma_{3}^{+}:=\frac{1}{n} \sum_{i=1}^{n} h\left(\beta_{n i}\right) \mathbb{I}_{] D_{n}, \infty[}\left(h\left(\beta_{n i}\right)\right) \quad \text { and } \quad \Sigma_{3}^{-}:=\frac{1}{n} \sum_{i=1}^{n} h\left(\beta_{n i}\right) \mathbb{I}_{]-\infty,-D_{n}[}\left(h\left(\beta_{n i}\right)\right) \text {. }
$$


In accordance with (3.21), there exists $c>0$ such that

$$
\Sigma_{3}^{+} \leq \Lambda_{1}+\Lambda_{2},
$$

with the notation

$$
\Lambda_{1}:=\frac{C_{2}}{n} \sum_{i=1}^{n} \mathbb{I}_{] c n^{\delta-1 / \alpha} \cdot \tilde{\ell}(n) ;+\infty[}\left(n^{-1 / \alpha} \tilde{\ell}(n) \beta_{n i}\right)
$$

and

$$
\Lambda_{2}:=\frac{C_{1}}{n^{1-1 / \alpha} \cdot \tilde{\ell}(n)} \sum_{i=1}^{n}\left(n^{-1 / \alpha} \cdot \tilde{\ell}(n) \beta_{n i}\right) \mathbb{I}_{] c n^{\delta-1 / \alpha} \cdot \tilde{\ell}(n) ;+\infty[}\left(n^{-1 / \alpha} \tilde{\ell}(n) \beta_{n i}\right) .
$$

Since $\tilde{\ell}$ is a slowly varying function at $0^{+}$, for each $\varepsilon>0$ we have $n^{-\varepsilon}<\tilde{\ell}(n)<n^{\varepsilon}$ if $n$ is large enough. Choosing $\varepsilon>0$ such that $\delta-1 / \alpha-\varepsilon>0$, we get that

$$
c n^{\delta-1 / \alpha-\varepsilon}<c n^{\delta-1 / \alpha} \tilde{\ell}(n) .
$$

Consequently,

$$
\Lambda_{1} \leq \frac{C_{2}}{n} \sum_{i=1}^{n} \mathbb{I}_{11,+\infty[}\left(X_{n, i}\right)
$$

and

$$
\Lambda_{2} \leq \frac{C_{1}}{n^{1-1 / \alpha-\varepsilon}} \sum_{i=1}^{n}\left(X_{n, i}\right) \mathbb{I}_{] 1 ;+\infty[}\left(X_{n, i}\right) .
$$

The sum on the right-hand side of (3.26) is bounded by the number of jumps of $\xi$ greater than 1 and is therefore finite a.s. Indeed, it is easy to show that, for each $\varepsilon>0$,

$$
\limsup _{n} \sum_{i=1}^{n} \mathbb{I}_{[1+\varepsilon,+\infty)}\left(X_{n, i}\right) \leq N_{\varepsilon}
$$

where $N_{\varepsilon}$ is the number of jumps greater than $1+\varepsilon$. By similar arguments, the right-hand side of (3.27) can be bounded by the sum of jumps and is therefore finite. Thus $\Sigma_{3}^{+} \longrightarrow 0$ almost surely. The convergence $\Sigma_{3} \longrightarrow 0$ follows in the same way.

\subsection{Case $\sigma \neq 0$}

Suppose now that $\sigma \neq 0$ and $\gamma=0$. In view of decomposition (3.1) we are interested in the asymptotic behavior of $\xi(t)=\sigma W_{t}+Y_{t}$. Clearly, we can let $\sigma=1$ without loss of generality. It is shown in [8] that for each $t \in[0,1]$,

In addition, from the case $\sigma=0$ we deduce

$$
\frac{1}{\sqrt{n}} \mathrm{C}_{n}^{0}[W](t) \longrightarrow \mathrm{L}_{1}^{(2)}(t) \quad \text { a.s. }
$$

$$
\frac{1}{n^{1-\frac{1}{\alpha} \tilde{\ell}(n)}} \mathrm{C}_{n}^{0}[Y](t) \longrightarrow \mathrm{L}_{\alpha}^{(1)}(\mathrm{t}) \text { a.s. }
$$

when $n \rightarrow \infty$. Fix $t \in[0,1]$. We have to investigate $\frac{1}{\sqrt{n}} \mathrm{C}_{n}^{0}[W+Y](t)$ when $n \rightarrow \infty$. A well known property of operator $T$ is $\|T f-T g\|_{1} \leq\|f-g\|_{1}$ where $\|\cdot\|_{1}$ is the usual $L^{1}$-norm (cf. [8] for references). Observe also that $T\left(W_{n}+Y_{n}\right)^{\prime}=T\left(W_{n}^{\prime}+Y_{n}^{\prime}\right)$. These notes imply the equality

$$
\frac{1}{\sqrt{n}} \mathrm{C}_{n}^{0}[W+Y](t)=\frac{1}{\sqrt{n}} \int_{0}^{t} T\left(W_{n}^{\prime}+Y_{n}^{\prime}\right)(s) \mathrm{d} s
$$


and the bounds

$$
\begin{aligned}
\left|\frac{1}{\sqrt{n}} \int_{0}^{t} T\left(W_{n}^{\prime}+Y_{n}^{\prime}\right)(s) \mathrm{d} s-\frac{1}{\sqrt{n}} \int_{0}^{t} T\left(W_{n}^{\prime}\right)(s) \mathrm{d} s\right| & \leq \frac{1}{\sqrt{n}} \int_{0}^{1}\left|T\left(W_{n}^{\prime}+Y_{n}^{\prime}\right)(s)-T W_{n}^{\prime}(s)\right| \mathrm{d} s \\
& \leq \frac{1}{\sqrt{n}} \int_{0}^{1}\left|Y_{n}^{\prime}(s)\right| \mathrm{d} s=n^{1-\frac{1}{\alpha}-\frac{1}{2}} \tilde{\ell}(n) \cdot \frac{1}{n^{1-\frac{1}{\alpha}} \tilde{\ell}(n)} \int_{0}^{1}\left|Y_{n}^{\prime}(s)\right| \mathrm{d} s .
\end{aligned}
$$

From Lemma 4.2 we have that $\frac{1}{n^{1-\frac{1}{\alpha}} \tilde{\ell}(n)} \int_{0}^{1}\left|Y_{n}^{\prime}(s)\right| \mathrm{d} s$ converges to $\int_{0}^{1}\left|F_{\alpha}^{-1}(s)\right| \mathrm{d} s$. Since $1<\alpha<2$, this implies that $\frac{1}{n^{1-\frac{1}{\alpha}} \tilde{\ell}(n)} \int_{0}^{1}\left|Y_{n}^{\prime}(s)\right| \mathrm{d} s$ is finite $a . s$. and also that $n^{1-\frac{1}{\alpha}-\frac{1}{2}} \tilde{\ell}(n)$ tends to zero. Hence, the last inequality above shows that, for each $t \in[0,1]$,

$$
\left|\frac{1}{\sqrt{n}} \int_{0}^{t} T\left(W_{n}^{\prime}+Y_{n}^{\prime}\right)(s) \mathrm{d} s-\frac{1}{\sqrt{n}} \int_{0}^{t} T\left(W_{n}^{\prime}\right)(s) \mathrm{d} s\right| \longrightarrow 0 \quad \text { a.s. }
$$

when $n \rightarrow \infty$. The proof of Theorem 2.1 is now complete.

Acknowledgements. The authors are grateful to Ricardas Zitikis for helpful discussions and precious remarks about the topic of this paper and to Michel Lifshits for his interest in this work. The authors wish also to thank the referees for their very careful reading of this manuscript and for making a number of very useful suggestions.

\section{REFERENCES}

[1] J-M. Azaïs and M. Wschebor, Almost sure oscillation of certain random processes. Bernoulli 2 (1996) 257-270.

[2] J. Bertoin, Lévy processes. Cambridge University Press (1998).

[3] N.J. Bingham, C.M. Goldie and J.L. Teugels, Regular variation. Cambridge University Press (1987).

[4] M. Csörgö, J.L. Gastwirth and R. Zitikis, Asymptotic confidence bands for the Lorenz and Bonferroni curves based on the empirical Lorenz curve. J. Statistical Planning and Inference 74 (1998) 65-91.

[5] M. Csörgö and R. Zitikis, On confidence bands for the Lorenz and Goldie curves, in Advances in the theory and practice of statistics. Wiley, New York (1997) 261-281.

[6] M. Csörgö and R. Zitikis, On the rate of strong consistency of Lorenz curves. Statist. Probab. Lett. 34 (1997) 113-121.

[7] M. Csörgö and R. Zitikis, Strassen's LIL for the Lorenz curve. J. Multivariate Anal. 59 (1996) 1-12.

[8] Y. Davydov, Convex rearrangements of stable processes. J. Math. Sci. 92 (1998) 4010-4016.

[9] Y. Davydov and V. Egorov, Functional limit theorems for induced order statistics. Math. Methods Stat. 9 (2000) $297-313$.

[10] Y. Davydov, D. Khoshnevisan, Zh. Shi and R. Zitikis, Convex Rearrangements, Generalized Lorenz Curves, and Correlated Gaussian Data. J. Statistical Planning and Inference 137 (2006) 915-934.

[11] Y. Davydov and E. Thilly, Convex rearrangements of Gaussian processes. Theory Probab. Appl. 47 (2002) $219-235$.

[12] Y. Davydov and E. Thilly, Convex rearrangements of smoothed random processes, in Limit theorems in probability and statistics. Fourth Hungarian colloquium on limit theorems in probability and statistics, Balatonlelle, Hungary, June 28-July 2, 1999. Vol I. I. Berkes et al., Eds. Janos Bolyai Mathematical Society, Budapest (2002) 521-552.

[13] Y. Davydov and A.M. Vershik, Réarrangements convexes des marches aléatoires. Ann. Inst. Henri Poincaré, Probab. Stat. 34 (1998) 73-95.

[14] Y. Davydov and R. Zitikis, Generalized Lorenz curves and convexifications of stochastic processes. J. Appl. Probab. 40 (2003) 906-925.

[15] Y. Davydov and R. Zitikis, Convex rearrangements of random elements, in Asymptotic Methods in Stochastics. American Mathematical Society, Providence, RI (2004) 141-171.

[16] R.A. Doney and R.A. Maller, Stability and attraction to normality for Lévy processes at zero and at infinity. J. Theor. Probab. 15 (2002) 751-792.

[17] W. Feller, An introduction to probability theory and its applications, Vol. I and II. John Wiley and Sons Ed. (1968).

[18] I.I. Gihman and A.V. Skorohod, Introduction to the theory of random processes. W. B. Saunders Co., Philadelphia, PA (1969).

[19] W. Linde, Probability in Banach Spaces - Stable and Infinitely Divisible Distributions. Wiley, Chichester (1986).

[20] A. Philippe and E. Thilly, Identification of locally self-similar Gaussian process by using convex rearrangements. Methodol. Comput. Appl. Probab. 4 (2002) 195-209.

[21] B. Ramachandran, On characteristic functions and moments. Sankhya 31 Series A (1969) 1-12.

[22] M. Wschebor, Almost sure weak convergence of the increments of Lévy processes. Stochastic Proc. App. 55 (1995) $253-270$.

[23] M. Wschebor, Smoothing and occupation measures of stochastic processes. Ann. Fac. Sci. Toulouse, Math 15 (2006) 125-156. 